\title{
ON THE FLOW PAST RECTANGULAR CYLINDERS: PHYSICAL ASPECTS AND NUMERICAL SIMULATION
}

\begin{abstract}
O. Almeida
S. S. Mansur

and A. Silveira-Neto ${ }^{c}$

${ }^{a}$ EMBRAER S.A.

Aeroacústica / Sistemas de Propulsão

São José dos Campos, SP, Brasil

odenir.almeida@embraer.com.br

${ }^{\mathrm{b}}$ Universidade Estadual Paulista - UNESP

Departamento de Engenharia Mecânica

Ilha Solteira, SP, Brasil

mansur@dem.feis.unesp.br

ABSTRACT

This paper concerns with unsteady incompressible flows around rectangular cylinders with side ratio varying from 1 to 10 . Phenomenological aspects are discussed and numerical simulations are performed using a SIMPLEC finite volume code. A third-order QUICK scheme is employed for the advective terms in the Navier-Stokes equations, while a second-order fully implicit method is used for the time discretization. For validation purpose, preliminary simulations are carried out at $R e=300$. Afterwards, the flow patterns and the wake periodic features are examined at $R e=1,000,5,000$, and 22,000, for which turbulent effects should not be neglected. In some of those cases, large-eddy simulation (LES) is employed, using the classical sub-grid Smagorinsky model. Important physical mechanisms determining vortex shedding frequency are placed in evidence. The present predictions are compared with numerical and experimental results from other works and a good agreement is reached.
\end{abstract}

${ }^{\mathrm{c}}$ Universidade Federal de Uberlândia - UFU

Faculdade de Engenharia Mecânica

Uberlândia, MG, Brasil

aristeus@mecanica.ufu.br
Keywords: External flow, rectangular cylinder, aspect ratio, large eddy simulation.

\section{NOMENCLATURE}

A

$B$

$C_{S}$

d

D

$f$

$k$ pressure, $\mathrm{Pa}$ time, $\mathrm{s}$ time step, $\mathrm{s}$ aspect ratio rectangular cylinder streamwise length, $m$ rectangular cylinder height, $\mathrm{m}$

Smagorinsky constant normal distance to the closest wall, $\mathrm{m}$ characteristic length, $\mathrm{m}$ vortex shedding frequency, $\mathrm{s}^{-1}$ von Kármán constant subgrid characteristic length, $\mathrm{m}$ rectangular cylinder spanwise length, $\mathrm{m}$ number of grids over the cylinder side

Reynolds number rate-of-strain tensor, $\mathrm{s}^{-1}$ Strouhal number $x_{i}$-component velocity, $\mathrm{m} \cdot \mathrm{s}^{-1}$ inlet velocity, $\mathrm{m} . \mathrm{s}^{-1}$ spatial coordinates, $\mathrm{m}$ kinematic viscosity, $\mathrm{m}^{2} . \mathrm{s}^{-1}$ molecular viscosity, Pa.s eddy viscosity, Pa.s mass density, $\mathrm{kg} . \mathrm{m}^{-3}$ subgrid-scale Reynolds stress, $\mathrm{Pa}$ normal stress, $\mathrm{Pa}$ computational cell volume, $\mathrm{m}^{3}$ Kronecker delta dimensionless time step

\section{INTRODUCTION}

Flow around square and rectangular cylinders is a frequent happening in engineering. Tall buildings, monuments, and towers are permanently exposed to wind. Similarly, piers, bridge pillars, and legs of offshore platforms are continuously submitted to the load produced by maritime or fluvial streams. This kind of flow is also found in many other technical applications, especially those concerning with thermal and hydraulic devices. For that reason, the literature about this subject is relatively well supplied, including both experimental and numerical investigations. In spite of that, several aspects of this topic remain misunderstood.

Since long ago, experimental works have been conducted in wind tunnels or water channels to determinate aerodynamics loads on cylinders of different cross sections and vortex shedding frequency in its wakes - Vickery (1966), Nakaguchi et al. (1968), Bearman and Trueman (1972), Durão et al. (1988), Hasan (1989), Nakamura et al. (1991), Norberg (1993), Lyn et al. (1995), Reisenberger et al. (2004), and Venugopal et al. (2006). In some cases, measurements are combined with flow visualizations in order to provide phenomenological insight on the fluid motion at the vicinity of the solid body - Okajima (1982), Davis et al. (1984), Nakamura et al. (1996), Lindquist (2000), Mills et al. (2003), Ozgoren (2006), Kuroda et al. (2007), Luo et al. (2007).

Numerical simulation of such a flow, in turn, never was considered a trivial task. Indeed, 
notwithstanding the geometrical simplicity of the problem, flow surrounding a rectangular cylinder keeps a high level of complexity due to several factors: presence of adverse pressure gradients, separation and reattachment zones, recirculating regions, highly curved streamlines, and eddy interactions. Calculations performed before the nineties were basically $2 \mathrm{D}$ and, usually, for flows at moderate Reynolds numbers, in which the turbulent effects could safely be neglected - Fromm and Harlow (1963), Davis and Moore (1982), Lee and Leal (1986). Later on, following computer evolution, a number of studies came up that, by means of mesh refining, achieved higher Reynolds numbers, still using 2D approach - Okajima (1990), Ohya et al. (1992), Kondo and Yamada (1995), Tamura et al. (1995), Nakamura et al. (1996), Yu and Kareem (1998), Bouris and Bergeles (1999), Shimada and Ishihara (2002). Nowadays, due to considerable advances in computer technology and numerical methods, 3D simulations of the flow around rectangular cylinders are yet feasible, as it have been performed by Breuer and Pourquié (1996), Kogaki et al. (1997), Noda and Nakayama (2003), Sohankar (2006), Koobus et al. (2007), Kuroda et al. (2007).

According to Monkewitz and Nguyen (1987), the approach 2D suitably represent the main fluid motion properties, since the primary instability mode in the wake of a 2D bluff body is essentially 2D. Nevertheless, experimental studies by Szepessy and Bearman (1992) have established that $2 \mathrm{D}$ simulations may substantially overestimate the fluctuating loads. Thus, as important computational resources are available, adequate $3 \mathrm{D}$ calculations can offer detailed information about the flow, disclosing physical mechanisms which couldn't be identified in 2D simulations. On the other hand, it is wellknown that 2D simulations using refined grids frequently yield better results than $3 \mathrm{D}$ ones performed with coarser meshes. Moreover, in the lack of computational support appropriate to solve 3D flows, the 2D approach remains a profitable tool for fluid flow analysis.

In the present paper, some typical features of the unconfined flow around rectangular cylinders are reviewed and discussed in light of previous works. Thereafter, 2D numerical simulations are accomplished in order to investigate the incompressible flow around rectangular cylinders with aspect ratios varying between 1 and 10, and Reynolds number ranged from 300 to 22,000 . In some calculations, LES methodology has been employed, using the standard Smagorinsky sub-grid scale model. The results are discussed and confronted with numerical and experimental data from other authors.

\section{PHYSICAL CONSIDERATIONS AND GEOMETRICAL PARAMETERS}

Flow pattern of rectangular cylinders is intrinsically associated to the Reynolds number $(R e)$ and aspect ratio $(\phi)$, defined respectively as:
$R e=\frac{U_{\infty} B}{v}$

$\phi=A / B$

where $A, B$ and $U_{\infty}$ are represented in Fig. 1 .

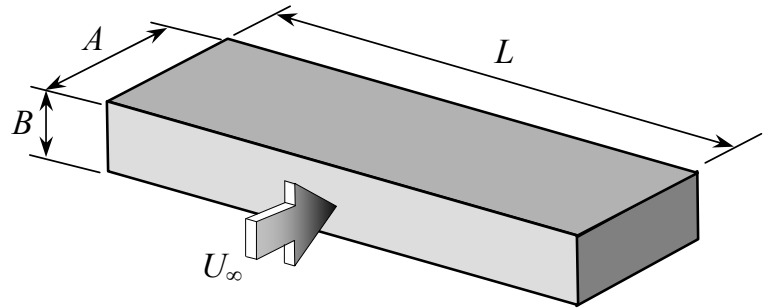

Figure 1. Rectangular cylinder in cross-flow.

Based on experimental visualizations by Lindquist (2000), Fig. 2 sketches different flow configurations over a rectangular cylinder at moderate Reynolds numbers. In the Fig. 2(a), a steady flow with symmetric streamlines is established around the whole body. In such a regime, flow detachment cannot be clearly identified even over the leading and trailing edges. As the freestream velocity increases, two recirculating bubbles appear at the cylinder rear - Fig. 2(b) -, which will become asymmetric as the subsequent Reynolds number increasing - Fig. 2(c). In that case, the shear layers downstream the cylinder become unstable and an oscillatory motion can be noted in the wake. The amplitude of the oscillations increases as the Reynolds number grow up - Fig. 2(d). In the next stage, shown in Fig. 2(e), Kelvin-Helmholtz instabilities are generated and advected downstream the cylinder. An alternating vortex shedding regime is then established creating the well-known von Kármán street. In Fig. 2(f), two recirculating bubbles can be identified on the leading edge vicinities due to flow separation at the sharp corners. If the cylinder side length is long enough, a flow reattachment will most likely occur over the two sides of the cylinder. The length of the reattachment region tends to increase as a function of the Reynolds number. Somewhere over the cylinder the shear layer breaks up originating a small discrete vortex that will be shed and advected towards the trailing edges - Fig. 2(g). For Reynolds number beyond 350 - Fig. 2(h) - vortex interactions and mixing in the wake are improved, eddy structures begin to lose their identity, and flow details may became harder to visualize.

In the periodic flow regimes, vortex shedding frequency $(f)$ is related to the mean velocity of the flow $\left(U_{\infty}\right)$ and to the body characteristic length $(D)$ through the Strouhal number $(S t)$, defined as:

$S t=f D / U_{\infty}$

where $D$ can be represented by the cylinder transversal height $B$ or the streamwise width $A$. 


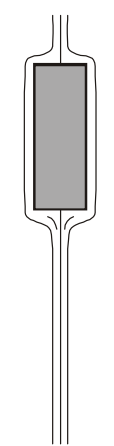

(a) $\operatorname{Re}<40$

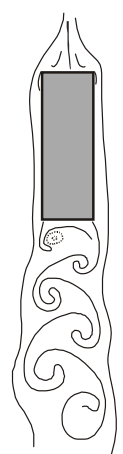

(e) $100<\operatorname{Re}<200$

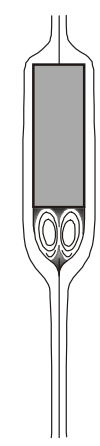

(b) $38<R e<46$



(f) $150<\operatorname{Re}<250$

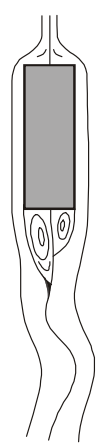

(c) $42<\operatorname{Re}<56$

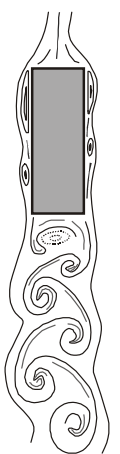

(g) $200<R e<350$

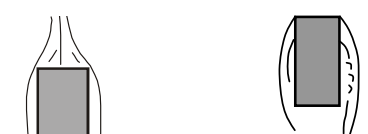

(a)





(b) $2.1<\phi \leq 6$ (c) $\phi>$

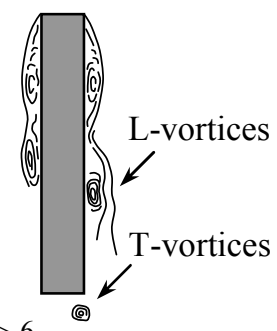

(2)

Figure 3. Influence of the aspect ratio on the flow configuration.

\section{MATHEMATICAL MODEL}

The flow is assumed to be incompressible, unsteady, and 2D, with constant fluid properties. Calculations have been accomplished in two different ways: a) by solving continuity and time-dependent Navier-Stokes equations without turbulence modeling, for relatively low Reynolds numbers; b) employing LES methodology associated with the standard Smagorinsky sub-grid scale model (Smagorinsky, 1963), for Reynolds numbers beyond 1,000 . In this latter case, the governing equations are obtained by means of a filtering process, which leads to the following simultaneous equation system:

$\frac{\partial \bar{u}_{j}}{\partial x_{j}}=0$

$\rho\left[\frac{\partial \bar{u}_{i}}{\partial t}+\frac{\partial}{\partial x_{j}}\left(\bar{u}_{i} \bar{u}_{j}\right)\right]=\frac{\partial}{\partial x_{j}}\left[\mu\left(\frac{\partial \bar{u}_{i}}{\partial x_{j}}+\frac{\partial \bar{u}_{j}}{\partial x_{i}}\right)\right]-\frac{\partial \bar{p}}{\partial x_{i}}-\frac{\partial \tau_{i j}}{\partial x_{j}}$

where the overbar denotes a filtered quantity and $\tau_{i j}$ is the subgrid-scale Reynolds stress, written as:

$\tau_{i j} \equiv \rho\left(\overline{u_{i} u_{j}}-\bar{u}_{i} \bar{u}_{j}\right)$

which appears as additional unknown, and require some kind of modeling to represent it. Classically, the closure process is performed using the Boussinesq hypothesis:

$\tau_{i j}=-2 \mu_{t} \bar{S}_{i j}+\frac{1}{3} \tau_{k k} \delta_{i j}$

where $\mu_{t}$ is the turbulent viscosity, $\delta_{i j}$ is the Kronecker delta, and $\bar{S}_{i j}$ is the rate-of-strain tensor for the resolved scales, which is calculated using the filtered velocity field:

$\bar{S}_{i j}=\frac{1}{2}\left(\frac{\partial \bar{u}_{i}}{\partial x_{j}}+\frac{\partial \bar{u}_{j}}{\partial x_{i}}\right)$

The turbulent viscosity is given by:

$\mu_{t}=\rho\left(C_{s} \ell\right)^{2}\left(2 \bar{S}_{i j} \bar{S}_{i j}\right)^{1 / 2}$

where $\ell$ is the characteristic length for sub-grid turbulent structure, whose value is computed using: 


$$
\ell=\min \left(k d, \vartheta^{1 / 3}\right)
$$

where $k=0,41$ is the von Kárman constant, $d$ is the normal distance to the closest wall, and $\vartheta$ is the local volume of the computational cell, i.e. $\vartheta=\Delta x_{1} \Delta x_{2}$ in 2D calculations.

The Smagorinsky constant $\left(C_{S}\right)$, analytically estimated by Lilly (1966) as being 0.18 for homogeneous isotropic turbulence, depends in fact on the flow features and the intrinsic characteristics of the computational code, and should be adjusted for each case by means of a calibration process. For flow past bluff bodies simulations the most common values found in the literature are ranged from 0.1 up to 0.2 . In this work, $C s$ has been adjusted to 0.1 by means of several preliminary tests and this value has been used in turbulent or transitional simulations.

\section{NUMERICAL PROCEDURE}

An incompressible SIMPLEC (Van Doormaal and Raithby, 1984) finite volume algorithm has been used, associated to QUICK scheme (Hayase et al. 1992) to treat the advective terms. A second-order central differences scheme has been employed for the diffusive terms. For time marching, a second-order fully implicit scheme has been adopted, with a dimensionless time step equal to:

$\Delta t^{*}=\Delta t U_{\infty} / B$

Figure 4 presents the computational domain, where a cylinder with aspect ratio equal to 7, placed at a distance of $X_{l u}$ downstream the inlet boundary, bisects horizontally the domain in two symmetric parts. A Cartesian grid, more refined close to the cylinder surface and in its wake, was employed. Around the cylinder, this mesh is uniformly distributed in both $x_{1}$ and $x_{2}$ directions, being non-uniform out of that region. The number of intervals over the lateral faces of the cylinder $(N)$ has been varied according to the aspect ratio factor $(\phi)$. In order to avoid numerical drawbacks, inside non-uniform zones the grid expansion factor has been limited to $1.2 \%$. Tables 1 and 2 summarize the main data concerning with the different meshes used in all the present simulations.

The boundary conditions have been prescribed as follows: a) at the upstream boundary, the inlet flow has been assumed to be uniform $\left(U_{\infty}\right)$; b) at the outlet, a zero gradient boundary condition for both $u_{1}$ and $u_{2}$ has been used; c) at the upper and lower boundaries, free stream boundary conditions have been set to represent a frictionless wall $\left(u_{2}=\partial u_{1} / \partial x_{2}=0\right)$; d $)$ inside the region occupied by the rectangular cylinder infinite viscosity has been adjusted, that is the same as imposing no-slip condition $\left(u_{1}=u_{2}=0\right)$ on its walls.

For the Strouhal number calculation, the temporal evolution of the $x_{2}$-component velocity has been captured with a numerical probe placed $1.5 B$ downstream the cylinder base and $2 B$ below the domain centerline. Two different time steps, $\Delta t=10^{-4} \mathrm{~s}$ and $\Delta t=10^{-3} \mathrm{~s}$, have been used to advance the temporal solution. The smaller one was chosen to simulate the turbulent flow at $R e=22,000$, while the larger time step was suitable at low and moderate Reynolds numbers.

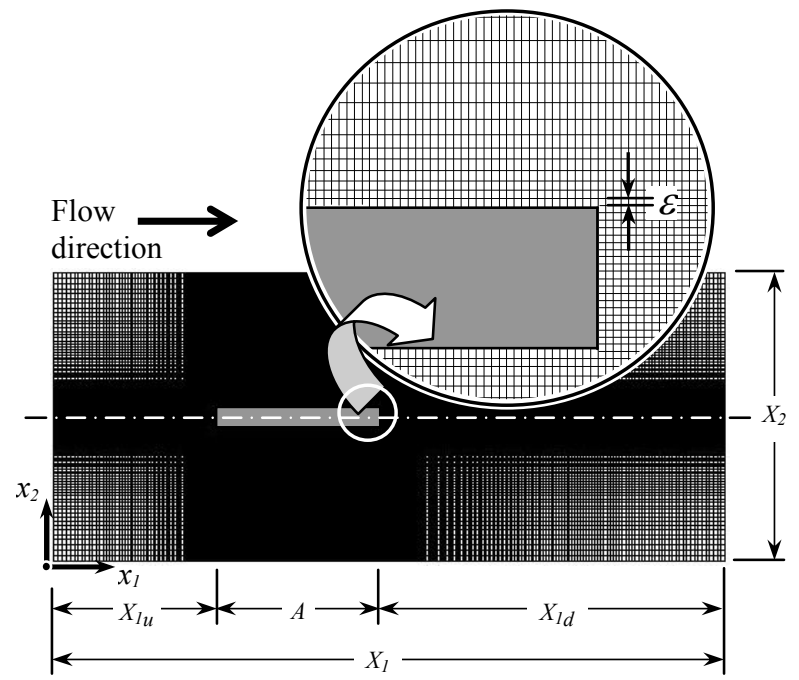

Figure 4. Computational domain and grid system for a cylinder with $\phi=7$.

Table 1. Computational domain configuration and grid size for $R e=300$.

\begin{tabular}{cccl}
\hline $\boldsymbol{\phi}$ & Mesh & $\boldsymbol{N} / \boldsymbol{A}$ & \multicolumn{1}{c}{$\boldsymbol{\varepsilon} / \boldsymbol{B}$} \\
\hline 5 & $250 \times 90$ & 100 & 0.0003 \\
7 & $270 \times 90$ & 120 & 0.00035 \\
9 & $290 \times 90$ & 140 & 0.000385 \\
10 & $300 \times 90$ & 150 & 0.0004 \\
11 & $310 \times 90$ & 160 & 0.0004125 \\
\hline$X_{2}=15 B$ & $(90$ horizontal grids) \\
$X_{I u}=10 B$ & (50 vertical grids) \\
$X_{l d}=20 B$ & (100 vertical grids) \\
\hline \multicolumn{5}{r}{}
\end{tabular}

Table 2. Computational domain and grid size for $R e=1,000$ and 5,000.

\begin{tabular}{|c|c|c|c|}
\hline$\phi$ & Mesh & $N / A$ & $\varepsilon / B$ \\
\hline 1 & $240 \times 160$ & $20 *$ & \multirow{18}{*}{0.00075} \\
\hline 2 & $260 \times 160$ & 40 & \\
\hline 3 & $280 \times 160$ & 60 & \\
\hline 4 & $300 \times 160$ & 80 & \\
\hline 5 & $320 \times 160$ & 100 & \\
\hline 5.2 & $324 \times 160$ & 104 & \\
\hline 5.4 & $328 \times 160$ & 108 & \\
\hline 5.6 & $332 \times 160$ & 112 & \\
\hline 5.8 & $336 \times 160$ & 116 & \\
\hline 6 & $340 \times 160$ & 120 & \\
\hline 7 & $360 \times 160$ & 140 & \\
\hline 8 & $380 \times 160$ & 160 & \\
\hline 8.2 & $384 \times 160$ & 164 & \\
\hline 8.4 & $388 \times 160$ & 168 & \\
\hline 8.6 & $392 \times 160$ & 172 & \\
\hline 8.8 & $396 \times 160$ & 176 & \\
\hline 9 & $400 \times 160$ & 190 & \\
\hline 10 & $420 \times 160$ & 200 & \\
\hline \multicolumn{3}{|c|}{$X_{2}=13 B$} & \\
\hline \multicolumn{3}{|c|}{$X_{1 u}=7 B$} & \\
\hline \multicolumn{3}{|c|}{$X_{I d}=15 B$} & \\
\hline
\end{tabular}

* N/A increased to 40 for square cylinder simulated at $R e=22,000$. 


\section{RESULTS AND DISCUSSION}

Preliminary simulations have been carried out in order to define adequate computational domains to assure grid independence. Both uniform and non-uniform meshes have been tested, with different refinements.

The first definitive simulations were performed without turbulence modeling, for rectangular cylinders with $5 \leq \phi \leq 11$ and $R e=300$. The principal physical goal here was to characterize the flow structure around the cylinder, as well as to determinate the vortex shedding frequencies for each case considered, comparing the present predictions with data of other authors.

The periodic behavior of the wake for $\phi$ up to 16 is characterized in the Fig. 5, in terms of the curve Strouhal versus aspect ratio. The good agreement among results indicates that the numerical schemes, the domain size, the mesh resolution, and the other parameters adjusted have been adequately chosen to capture the complex wake behavior, characterized by abrupt changes in the Strouhal number.

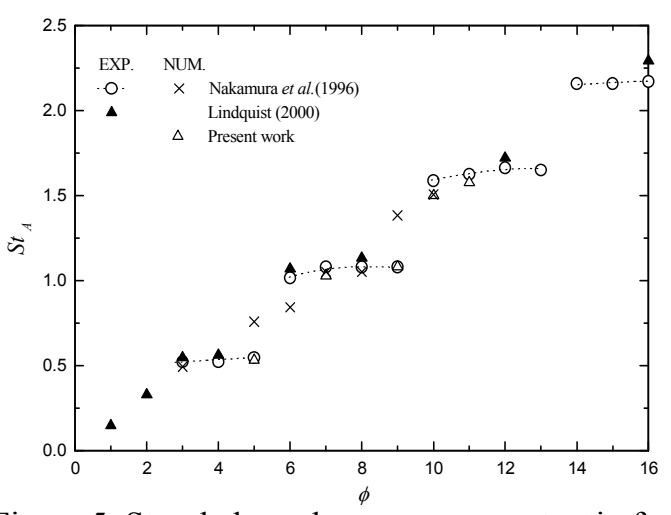

Figure 5. Strouhal number versus aspect ratio for cylinders with $1 \leq \phi \leq 16$, at $R e=300$.

The temporal evolution of streamlines over a cylinder with aspect ratio $\phi=7$ is depicted in Fig. 6, also for $R e=300$. It can be noted that the boundary layers on both cylinder sides detaches at the leading edges and reattaches after the middle of the body. Recirculating structures are identified close to the leading edges, over the cylinder sides, and at the rear of the body. The L-vortex and T-vortex discussed by Ozono et al. (1992) have been clearly captured in the present calculation.

In order to check the LES methodology implementation, a high Reynolds number calculation has been performed taking into account the flow around a square cylinder $(\phi=1)$. The mean longitudinal velocity and the total kinetic energy distribution at the centerline are presented in Fig. 7, for $R e=22,000$. In a general way, strong discrepancies among results of different authors are observed. Nevertheless, one can be verified that the present $2 \mathrm{D}$ calculation using LES is in a better agreement with the experimental data of Lyn et al. (1995) than several others, including the 3D LES of Murakami et al. (1993). At first, this would not be expected, since at $R e=22,000$ the three dimensional effects in the wake are very important, and a 3D simulation should generate a better representation of the flow. That worsened performance may be attributed to either insufficient mesh resolution or the numerical methodology used by those authors. It is interesting also to observe that the $k-\varepsilon$ simulations were not able to predict neither the length of the recirculation zone in the rear of the cylinder nor the velocity recovery in the wake.

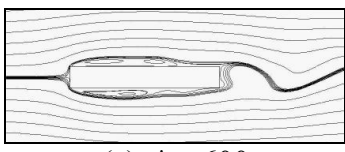

(a) $t^{*}=600$

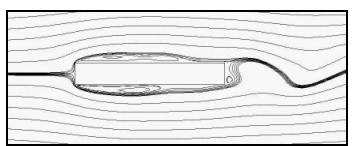

(b) $t^{*}=610$

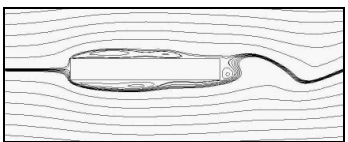

(c) $t^{*}=620$

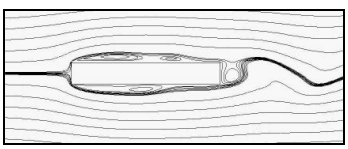

(d) $t^{*}=630$

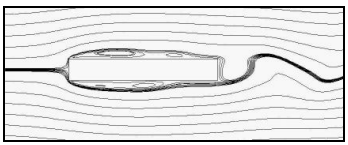

(e) $t^{*}=640$

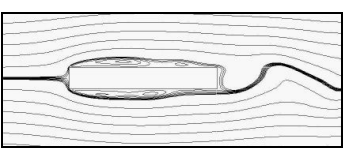

(f) $t^{*}=650$

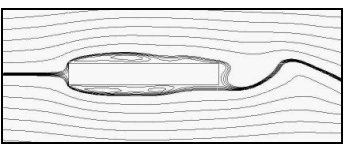

(g) $t^{*}=660$

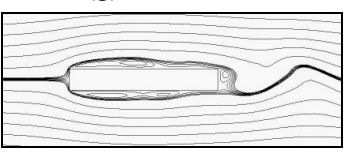

(h) $t^{*}=670$



(i) $t^{*}=680$

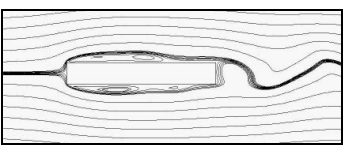

(j) $t^{*}=690$
Figure 6. Temporal evolution of the streamlines contours for a cylinder with $\phi=7$, at $R e=300$.

The last part of this paper is devoted to the simulation of transitional flows around rectangular cylinders. As a first approach, calculations were performed without turbulence modeling for cylinders with $\phi=1,5$, and 10, at $\operatorname{Re}=1,000$. Figure 8 shows the typical $x_{2}$-velocity signals captured by numerical probes positioned behind the cylinders. The respective power spectral distributions, coming from a fast Fourier transform (FFT) of those fluctuating signals, are also presented, allowing to determine the dimensionless vortex shedding frequencies $(S t)$, which correspond to the picks of the larger amplitude founded in each graph.

It is important to mention that the broad range of frequencies that characterizes some of those spectra may not have physical meaning, since no turbulence model has been used and the molecular viscosity alone could be insufficient to dissipate adequately the kinetic energy of the flow.

Hence, similar calculations have been accomplished at the same precedent conditions using LES methodology with Smagorinsky sub-grid model. As expected, the results presented in Fig. 9 show that 




(a) Mean horizontal velocity.

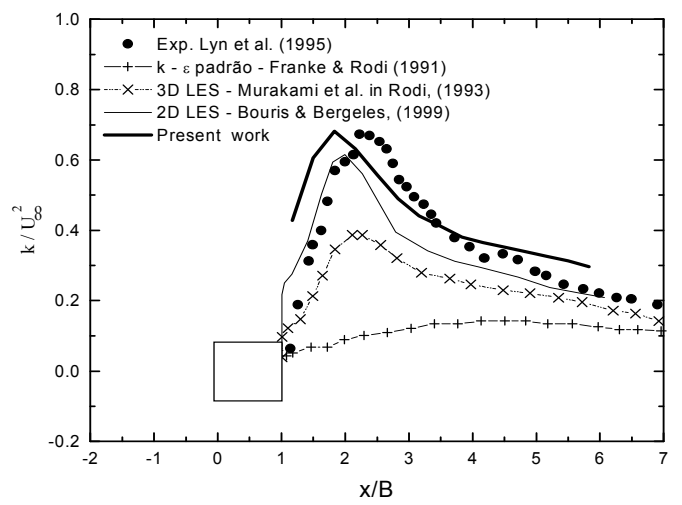

(b) Kinetic energy.

Figure 7. Mean horizontal velocity and total kinetic energy distribution along the cylinder centerline, at $R e=22,000$.

the velocity signals are sharply more organized than those previously obtained. Consequently, their corresponding Fourier transforms present only some few discrete peaks, indicating that LES methodology introduced a damping effect on the flow, eliminating part of the spurious instabilities present in the prior signals.

Other simulations have been executed for cylinders with $1 \leq \phi \leq 16$. The results are summarized in Fig. 10 and 11 in terms of St versus $\phi$, for $R e=1,000$ and 5,000, respectively. The present predictions are compared with results from Nakamura et al. (1996) and Lindquist (2000), for $R e=1,000$, and Okajima (1982) and Okajima (1991), for $R e=5,000$. For both flow conditions a good agreement has been found.

As already observed in the simulations for $R e=300$, presented in Fig. 5, the Strouhal number increases with the aspect ratio up to certain value and then shifts abruptly towards a higher value, presenting a very stiffen behavior in a specific value of $\phi$. Seemingly, this trend repeats itself in intervals that contain three integer aspect ratios. The dashed lines in Fig. 10 and 11 point out that trend in the Strouhal number curves. Although this singular behavior has been also captured in simulations without turbulence model, the LES results are in better agreement with experimental data. (a) $\phi=1$
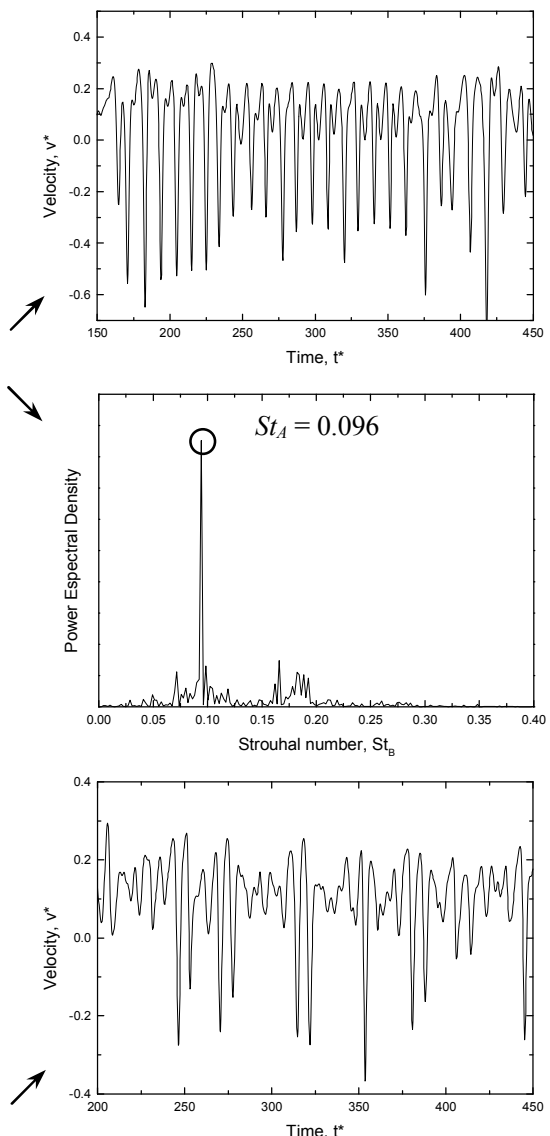

(b) $\phi=5$

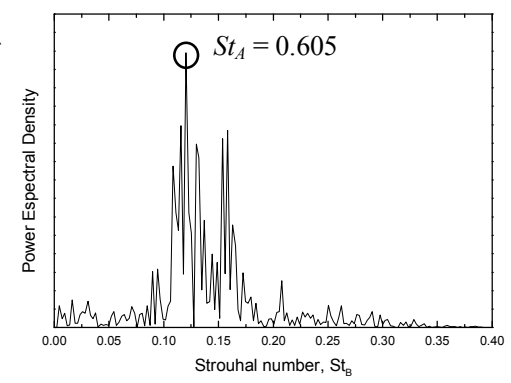

(c) $\phi=10$
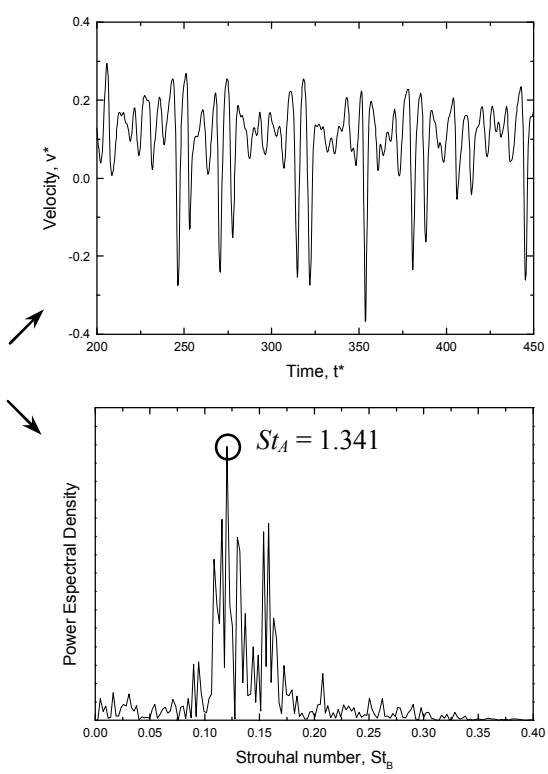

Figure 8. Velocity signals and respective Fourier transforms for different cylinders, at $R e=1,000$. 
(a) $\phi=1$
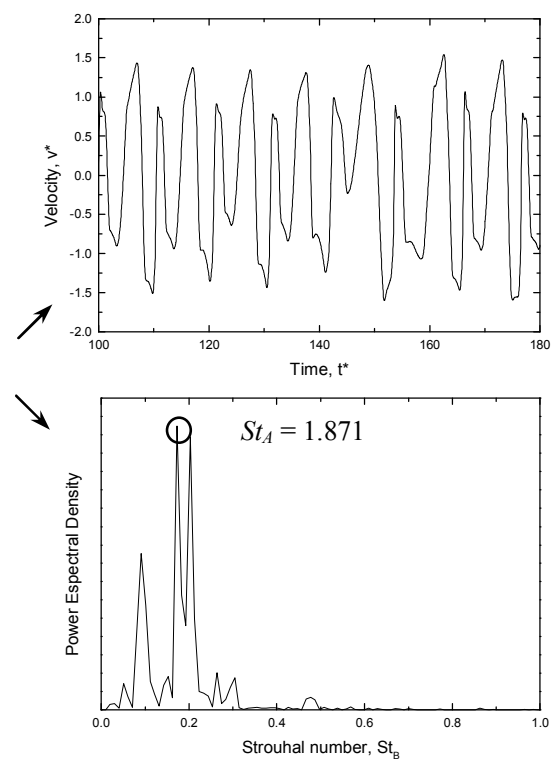

(b) $\phi=5$
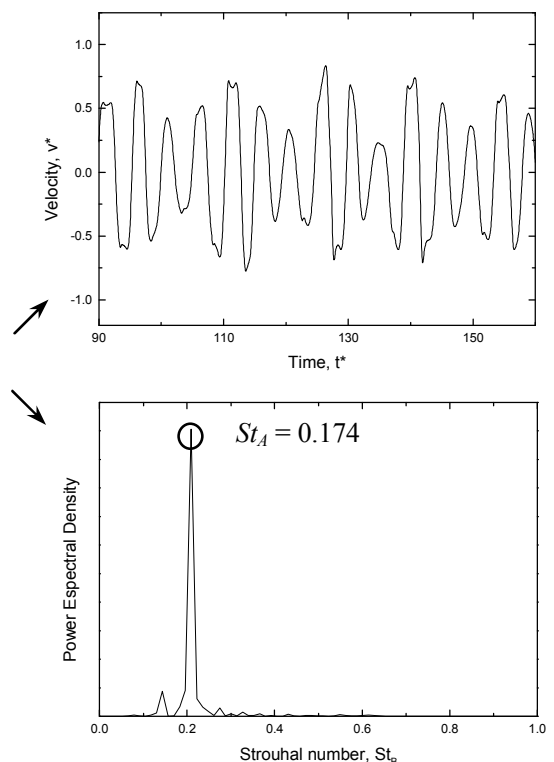

(c) $\phi=10$


Figure 9. Velocity signals and respective Fourier transforms for different cylinders, at $R e=1,000$, using LES.

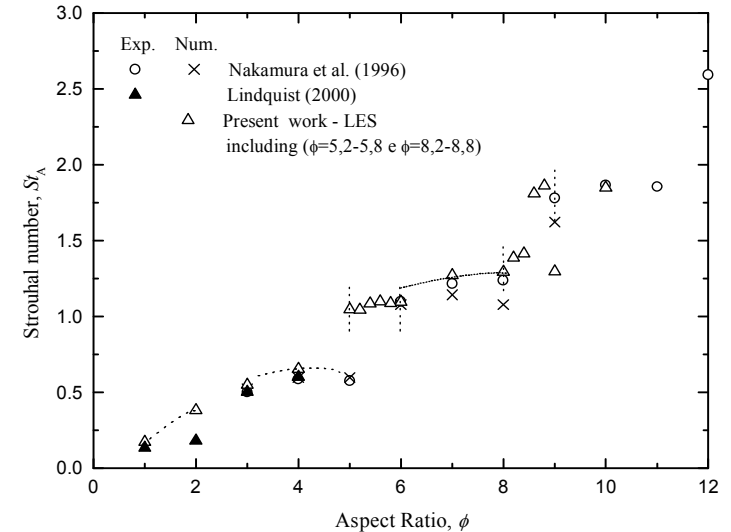

Figure 10. Strouhal number versus aspect ratio for different cylinders, at $R e=1,000$.

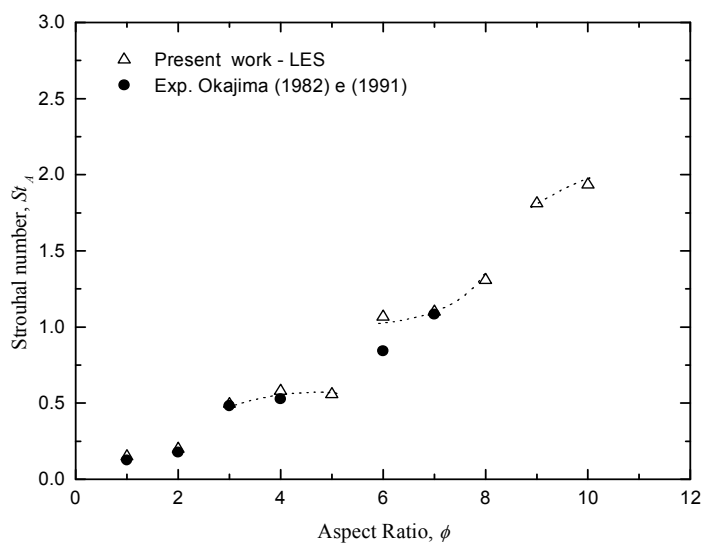

Figure 11. Strouhal number versus aspect ratio for different cylinders, at $R e=5,000$.

Physically, those stepwise discontinuities, or plateaus, are related to a transition in the vortex shedding mode, as described in details by Nakamura et al. (1996) and Lindquist (2000). Briefly, for Reynolds numbers higher than 250, the vortex shedding from rectangular cylinders is influenced by the so-called impinging-shear-layer instability, or simply ISLI. Differently from von Kármán instabilities, where two separated shear layers interact between themselves at the trailing edges, by means of ISLI mechanism a single separated shear layer can became unstable in the presence of a sharp trailing edge corner, as well explained by Ohya et al. (1992). As a consequence of that, the recirculation bubbles formed over the cylinder become unstable and begin to wobble and split in alternated discrete vortices, which are detached and advected downstream of the obstacle.

The numerical visualization of the vorticity field is very useful for the understanding of the Strouhal number behavior. Figures 12, 13, and 14 show the isovorticity contours related to the flow over the cylinders having $\phi=4,7$, and 10 , respectively, using LES. The time sequence shows the flow evolution, illustrating the dynamics of vortex formation and advection over and downstream the cylinder. It can be noted that the L-vortices formed on the faces of the cylinder are advected downstream and interact 
with the T-vortices formed in the rear of the cylinder. This interaction seems to be stronger as the aspect ratio $(\phi)$ is increased.

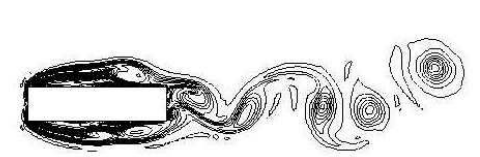

(a) $t^{*}=166,6$

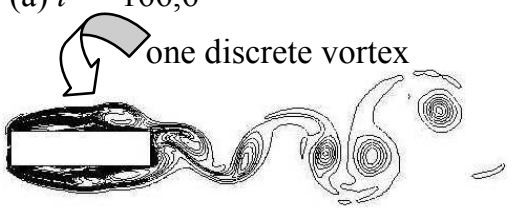

(b) $t^{*}=167,2$
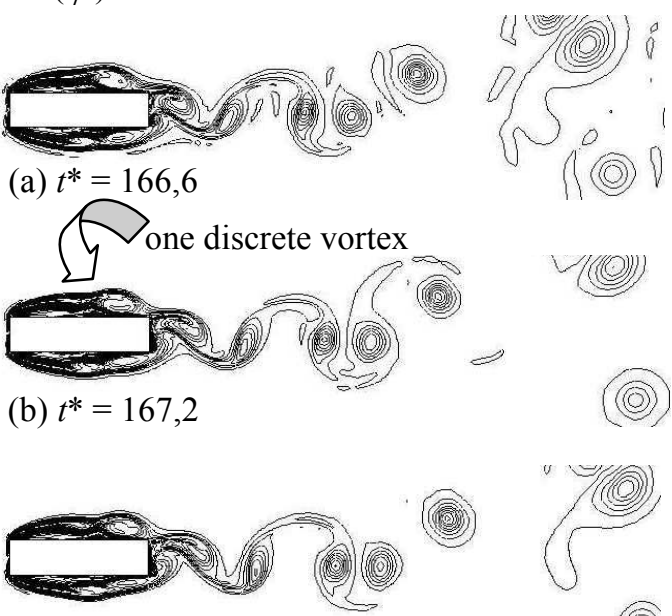

(c) $t^{*}=167,8$

Figure 12. Temporal evolution of the isovorticity contours for a cylinder with $\phi=4$, at $R e=1,000$, using LES.

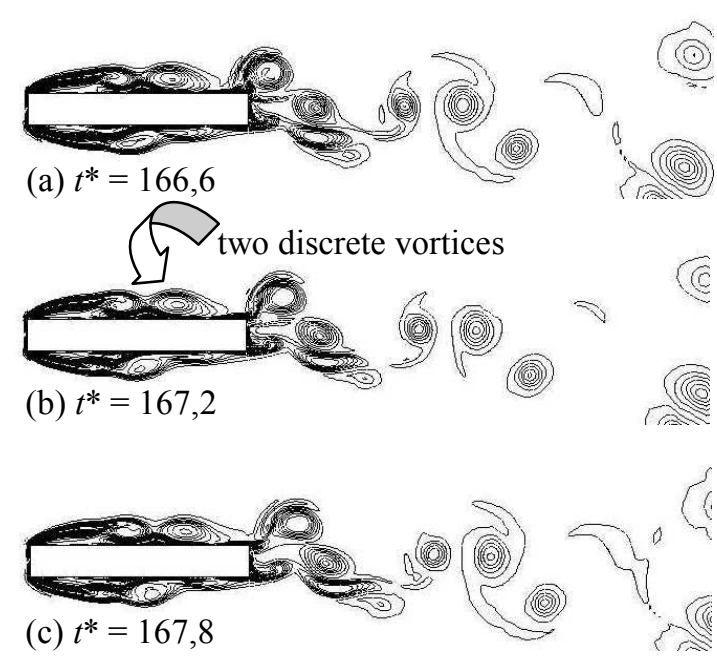

Figure 13. Temporal evolution of the isovorticity contours for a cylinder with $\phi=7$, at $R e=1,000$, using LES.


Figure 14. Temporal evolution of the isovorticity contours for a cylinder with $\phi=10$, at $R e=1,000$, using LES.
The amount of vortices $(n)$ observed over the cylinder face and the Strouhal number $\left(S t_{A}\right)$, based on streamwise width $A$, can be correlated by the following empiric expression, due to Ohya et al. (1992) and Nakamura et al. (1996):

$S t_{A} \cong 0.6 n$

As indicated in the isovorticity maps of those figures, $n=1, n=2$, and $n=3$ for cylinders with $\phi=4, \phi=7$, and $\phi=10$, respectively. Using Eq.(11), the Strouhal number assumes values of $0.6,1.2$, and 1.8 , corresponding to the three first plateaus shown in Fig. 10.

In order to understand the physical behavior of the vortex shedding process related to the jump that appears in the Strouhal number, isovorticity contours of the vortex wake for cylinders with $5.2 \leq \phi \leq 5.8$ and $8.2 \leq \phi \leq 8.8$ are shown in Fig. 15 and 16 respectively. The number of vortices over the cylinders will determine the fundamental frequency of vortex shedding.

In the Fig. 15 there are only two vortices over the cylinders and the Strouhal numbers are nearly constant, keeping the Strouhal number at a same plateau, as seen in the Fig. 10. In Fig. 16 it can be identified two galloping vortices over the cylinders with $\phi=8.2$ and $\phi=8.4$. Therefore, the Strouhal number is placed now in the second plateau. The appearance of a third vortex over the cylinders for $\phi=8.6$ results in an increasing of the Strouhal number, bringing it to the third plateau. So, it seems that the stiffen variation of the Strouhal number is strongly related to the number of vortices resident



(a) $\phi=5.2$

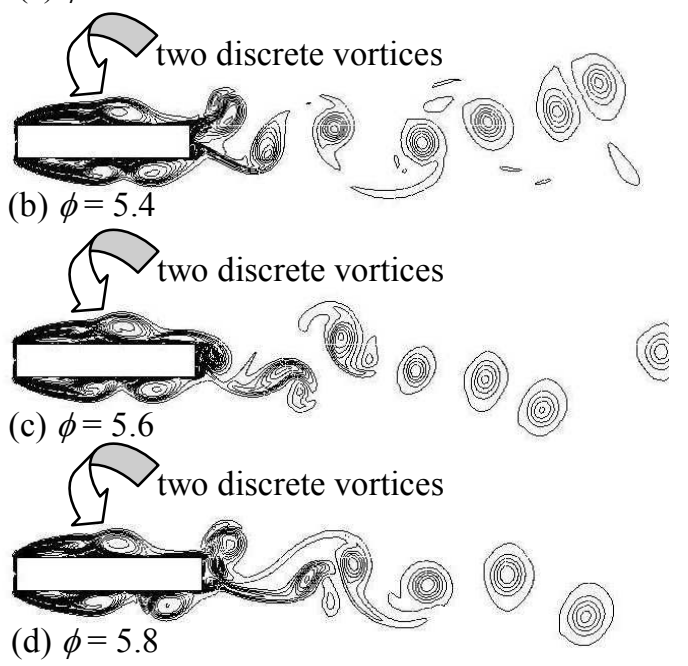

Figure 15 . Isovorticity contours for cylinders with $5.2 \leq \phi \leq 5.8$, at $R e=1,000$, using LES results. 




Figure 16. Isovorticity contours for cylinders with $8.2 \leq \phi \leq 8.6$, at $R e=1,000$, using LES.

over and under the cylinder. It should be remarked that this behavior is identified by two procedures: the FFT of the temporal velocity signature obtained by a numerical probe and by application of the Eq.(11) over the flow visualization given by vorticity fields.

\section{CONCLUDING REMARKS}

A numerical investigation of the flow around rectangular cylinders with aspect ratios varying from 1 to 10 has been presented. Two-dimensional calculations have been accomplished employing large eddy simulation with the classical Smagorinsky turbulence model. Some simulations without turbulence modeling have been also performed. The simulations allowed observe the vortex generation process over the cylinder and its wake. The effects of the rectangular cylinder aspect ratio on the wake dynamics have been investigated with the help of isovorticity visualizations, spectral analysis, and physical parameters calculations as Strouhal number. The isovorticity flow visualizations have also provided support to explain some quantitative results, such as the ISLI (impinging-shear-layer instability). In spite of the 2D calculations, and the transitional nature of these flows, the numerical results with LES present a good agreement with the experimental data presented in previous works. Also, LES exhibits much better results as compared with the calculation without turbulence modeling.

\section{ACKNOWLEDGMENTS}

The authors are grateful to the research agencies FAPESP, CAPES, and CNPq for the financial support granted to this work.

\section{REFERENCES}

Bearman, P. W. and Trueman, D. M., 1972, An Investigation of the Flow around Rectangular Cylinders, Aeronautic Quarterly, Vol. 23, pp. 229-237.
Bouris, D. and Bergeles, G., 1999, 2D LES of vortex shedding from a square cylinder, J. Wind Eng. and Ind. Aerodyn., Vol. 80, pp. 31-46.

Breuer, M. and Pourquié, M., 1996, First Experiences with LES of Flows Past Bluff Bodies, Proc. 3rd Int. Symp. of Engineering Turbulence Modelling and Measurements, Herakilon-Crete, Greece, pp. 177-186.

Davis, R. W. and Moore, E. F., 1982, A Numerical Study of Vortex Shedding from Rectangles, J. Fluid Mech., Vol. 116, pp.475-506.

Davis, R. W., Moore, E. F. and Purtell, L. P., 1984, A Numerical-Experimental Study of Confined Flow around Rectangular Cylinders, Phys. Fluids, Vol. 27, pp. 46-59.

Durão, D. F. G., Heitor, M. V. and Pereira, J. C. F., 1988, Measurements of Turbulent and Periodic Flows around a Square Cross-Section Cylinder, Exp. in Fluids, Vol. 6, pp. 298-304.

Fromm, J. E. and Harlow, F. H., 1963, Numerical Solution of the Problem of Vortex Street Development, Phys. Fluids, Vol. 6, pp. 975-982.

Franke, R. and Rodi, W., 1991. Calculation of Vortex Shedding Past a Square Cylinder with Various Turbulence Models, Proc. 8th Symp. on Turbulent Shear Flows, Munich.

Hasan, M. A. Z., 1989, The Near Wake Structure of a Square Cylinder, Int. J. Heat and Fluid Flow, Vol. 10, pp. 339-348.

Hayase, T., Humphrey, J. A. C. and Greif, R., 1992, A Consistently Formulated QUICK Scheme for Fast and Stable Convergence Using Finite-Volume Iterative Calculation Procedures, J. Comput. Physics, Vol. 98, pp. 108-118.

Kogaki, T., Kobayashi, T. and Taniguchi, N., 1997, Large Eddy Simulation of the Flow around a Rectangular Cylinder, Fluid Dynamics Research, Vol. 20, pp. 11-24.

Kondo, N. and Yamada, S., 1995, Third-Order Upwind Finite Element Computation of the Incompressible Navier-Stokes Equations. Part I: Computation of Flow around Rectangular Cylinders, Comput. Methods Appl. Mech. Eng., Vol. 127, pp. 87-97.

Koobus, B., Camarri, S., Salvetti, M. V., Wornom, S. and Dervieux, A., 2007, Parallel Simulation of Three-Dimensional Complex Flows: Application to Two-Phase Compressible Flows and Turbulent Wakes, Adv. Eng. Software, Vol. 38, pp. 328-337.

Kuroda, M., Tamura, T. and Suzuki, M., 2007, Applicability of LES to the Turbulent Wake of a Rectangular Cylinder - Comparison with PIV Data, J. Wind Eng. and Ind. Aerodyn., Vol. 95, pp. 1242-1258.

Lee, S.H. and Leal, L.G., 1986, Low-ReynoldsNumber Flow Past Cylindrical Bodies of Arbitrary CrossSectional Shape, J. Fluid Mech., Vol. 164, pp. 401-427.

Lilly, D.K., 1966, On the Application of the Eddy Viscosity Concept in the Inertial Subrange of 
Turbulence, NCAR Manuscript 123.

Lindquist, C., 2000, Experimental Study of the Flow around Square and Rectangular Cylinders (In Portuguese), M.Sc. Thesis, Universidade Estadual Paulista - UNESP, Ilha Solteira, Brazil.

Luo, S. C., Tong, X. H. and Khoo, B. C., 2007, Transition Phenomena in the Wake of a Square Cylinder, J. Fluids and Struct., Vol. 23, pp. 227-248.

Lyn, D. A., Einav, S., Rodi, W. and Park, J. H., 1995, A Laser Doppler Velocimetry Study of Ensemble-Averaged Characteristic of the Turbulent Near Wake of a Square Cylinder, J. Fluid Mech., Vol. 304, pp. 285-319.

Mills, R., Sheridan, J. and Hourigan, K., 2003, Particle Image Velocimetry and Visualization of Natural and Forced Flow around Rectangular Cylinders, J. Fluid Mech., Vol. 478, pp. 299-323.

Monkewitz, P. A. and Nguyen, L. N., 1987, Absolute Instability in the Near-Wake of TwoDimensional Bluff Bodies, J. Fluids and Struct., Vol. 1, pp. 165-184.

Murakami, S., Rodi, W. Mochida, A., Sakamoto, S., 1993, "Large Eddy Simulation of Turbulent Vortex Shedding Flow Past 2D Square Cylinder", ASME FED Publicayions, Vol.162, pp.113-120.

Nakaguchi, H., Hashimoto, K. and Muto, S., 1968, An Experimental Study on Aerodynamic Drag of Rectangular Cylinders (In Japanese), J. Japan Soc. of Aeronl. and Space Sciences, Vol. 16, pp. 1-5.

Nakamura, Y. and Nakashima, M., 1986, Vortex Excitation of Prisms with Elongated Rectangular, $\mathrm{H}$ and T Cross-Sections, J. Fluid Mech., Vol. 163, pp. 227-230.

Nakamura, Y., Ohya, Y., Ozono, S. and Nakayama, R., 1996, Reproducibility of Flow Past Two-Dimensional Rectangular Cylinders in a Homogeneus Turbulent Flow by LES, J. Wind Eng. and Ind. Aerodyn., Vol. 65, pp. 301-308.

Nakamura, Y., Ohya, Y. and Tsuruta, H., 1991, Experiments on vortex shedding from flat plates with square leading and trailing edges, J. Fluid Mech., Vol. 222, pp. 437-447.

Noda, H. and Nakayama, A., 2003, Reproducibility of Flow Past Two-Dimensional Rectangular Cylinders in a Homogeneus Turbulent Flow by LES, J. Wind Eng. and Ind. Aerodyn., Vol. 91, pp. 265-278.

Norberg, C., 1993, Flow around Rectangular Cylinders: Pressure Forces and Wake Frequencies, J. Wind Eng. and Ind. Aerodyn., Vol. 49, pp. 187-196.

Ohya, Y., Nakamura, Y., Ozono, S., Tsuruta, H. and Nakayama, R., 1992, Numerical Study of Vortex Shedding from Flat Plates with Square Leading and Trailing Edges, J. Fluid Mech., Vol. 236, pp. 445-460.

Okajima, A., 1982, Strouhal Numbers of Rectangular Cylinders, J. Fluid Mech., Vol. 123, pp. 379-398.

Okajima, A., 1990, Numerical Simulation of Flow around Rectangular Cylinders, J. Wind Eng. and Ind. Aerodyn., Vol. 33, pp. 171-180.

Okajima, A., 1991, Change of Flow about an Elongated Rectangular Cylinder in a Range of Reynolds Numbers of 200 to $0.7 \times 10^{4}$, ASME Forum on Turbulent Flows, Vol. 112, pp. 107-113.

Okajima, A., Ueno, H. and Sakay, H., 1992, Numerical Simulation of Laminar and Turbulent Flows around Rectangular Cylinders, Int. J. Num. Methods in Fluids, Vol. 15, pp. 999-1012.

Ozgoren, M., 2006, Flow Structure in the Downstream of Square and Circular Cylinders, Flow Measurement and Instrumentation, Vol. 17, pp. 225235.

Ozono, S., Ohya, Y., Nakamura, Y. and Nakayama, R., 1992, Stepwise Increase in the Strouhal Number for Flows around Flat Plates, Int. J. Num. Methods in Fluids, Vol. 15, pp. 1025-1036.

Reisenberger, E., Meile, W., Brenn, G. and Müller, W., 2004, Aerodynamic Behaviour of Prismatic Bodies with Sharp and Rounded Edges, Exp. in Fluids, Vol. 37, pp. 547-558.

Rodi, W., 1993, On the Simulation of Turbulent Flow Past Bluff Bodies, J. Wind Eng. and Ind. Aerodyn., Vol. 46/47, pp. 3-19.

Shimada, K. and Ishihara, T., 2002, Application of a Modified k-e Model to the Prediction of Aerodynamic Characteristics of Rectangular CrossSection Cylinders, J. Fluids and Struct., Vol. 16, pp. 465-485.

Smagorinsky, J., 1963, General Circulation Experiments with Primitive Equations, Mon. Weather Rev., Vol. 91, pp. 2501-2514.

Sohankar, A., 2006, Flow over a Bluff Body from Moderate to High Reynolds Numbers Using Large Eddy Simulation, Comp. and Fluids, Vol. 35, pp. 1154-1168.

Szepessy, S. and Bearman, P. W., 1992, Aspect Ratio and Endplate Effects on Vortex Shedding from a Circular Cylinder, J. Fluid Mech., Vol. 234, pp. 191-217.

Tamura, T., Itoh, Y, Wada, A. and Kuwahara, K., 1995, Numerical Study of Pressure Fluctuations on a Rectangular Cylinder in Aerodynamic Oscillations, J. Wind Eng. and Ind. Aerodyn., Vol. 54/55, pp. 239250 .

Van Doormaal, J. P. and Raithby, G. D., 1984, Enhancements of the SIMPLE Method for Prediction Incompressible Fluid Flows, Num. Heat Transfer, Vol. 7, pp. 147-163.

Venugopal, V, Varyani, K. S. and Barltrop, N. D. P., 2006, Wave Force Coefficients for Horizontally Submerged Rectangular Cylinders, Ocean Engineering, Vol. 33, pp. 1669-1704

Vickery, B. J., 1966, Fluctuating Lift and Drag on a Long Cylinder of Square Cross Section in a Smooth and in a Turbulent Stream, J. Fluid Mech., Vol. 25, pp. 481-494.

Yu, D. and Kareem, A., 1998, Parametric Study of Flow around Rectangular Prisms Using LES, J. Wind Eng. and Ind. Aerodyn., Vol. 77/78, pp. 653-662. 